\title{
INVESTIGATING THE NATIONAL DIGITAL CADASTRAL DATABASE (NDCDB) DATA HANDLING WITHIN GIS APPLICATIONS
}

\author{
N.Z.Abdul Halim ${ }^{1,2}$, S.A. Sulaiman ${ }^{1}$, K. Talib ${ }^{1}$, Z.A Majeed ${ }^{2}$ \\ ${ }^{1}$ Center of Studies for Surveying Science and Geomatics, Faculty of Architecture, Planning and Survey, University Technology \\ Mara, Shah Alam, Malaysia - nurzurairah@gmail.com \\ ${ }^{2}$ Department of Survey and Mapping Malaysia, Kuala Lumpur, Malaysia - zainal.amajeed@ jupem.gov.my
}

KEYWORDS: National Digital Cadastral Database, Multi-case study, spatial analysis

\begin{abstract}
:
This paper explains the investigation carried out to understand NDCDB data handling within GIS applications. The method used was a case study replicated to five established GIS applications from different agencies, namely eKadasOnline, SKiP, iPlan, TM SmartMap and DBKL Interactive Portal. The case study was propositioned to the statements of; i) "Such methods of applying NDCDB are because user understands its characteristics", and ii) such methods of applying NDCDB are because users know how to adopt it." Cross-case comparison analysis was then conducted to identify rival findings and explanation building. Based on the evidence of the multi-case study, it was concluded that such methods of adopting NDCDB by the GIS applications administrators and developers were because most of them have a partial understanding of the NDCDB characteristics which led to NDCDB being adopted based on the method that they think were suitable. Recommendations are highlighted in this paper to rectify knowledgebased mistakes found in this study, that included; i) ensuring the NDCDB's cut-off-date; ii) utilises all existing NDCDB layers; iii) ensure to use the map projection parameters are the authorised and official value; iv) understand that the NDCDB utilises the GDM2000 datum with ITRF2000 epoch 2006; v) False Easting and North Easting can be discarded for geocentric datum; vi) adopting the built-in Geocentric Cassini map projection of GIS software is not advisable; and vii) obtaining relevant additional NDCDB layers. With the recommendations emplace, it is hoped the full potential of NDCDB can be tapped especially for multipurpose cadastre implementation including to ease spatial analysis. Data replication, exhaustion of resources, and reduce risks or costly investments made by decision makers, policy makers, developers or individuals can be avoided when NDCDB is fully optimised for spatial analysis.
\end{abstract}

\section{INTRODUCTION}

The National Digital Cadastral Database or NDCDB is described as the most spatially accurate database as far as the land-related database is the concern in Malaysia (Halim et al., 2018). Statistics from MACGDI (2017) have shown NDCDB is frequently downloaded or applied as base maps for spatial analysis purposes since its first introduction in 2010. Conversely, it should be noted that unlike the universally known survey-accurate DCDB, NDCDB is a survey-accurate database but explicitly stores cadastral survey dataset and with limited cadastre information except those essential for land registration. Nevertheless, it plays a significant role mainly when the concern of the spatial analysis involves the land and people relationship (Halim et al., 2017). However, unused or improper cadastral data handling for spatial analysis may lead to questionable and unreliable result (Chan, 2016). Ahmad (2014) found in his study that most public organisation's GIS implementations were run by based on user's effort and definition, and was not guided by expertise or GIS champions. Unaware of the consequences of improper data handling, spatial analysis results may be spurious and do not capture the full potential of accurate analysis that NDCDB can offer. The potentials being; NDCDB i) is the most survey-accurate database as far as the land-based database is concerned; ii) covers land parcels that have been final surveyed which can be linked to their rights, restrictions and responsibility; and iii) is readily available on a large scale platform (Jamil, 2017). Moreover, casual GIS developers may not possess the necessary skills or knowledge of data handling for spatial analysis since most free and open sourced GIS software are easy to use but can easily be misused too (M. R. Dale \& Fortin, 2014). Therefore a multi-case study was deemed necessary to investigate how NDCDB is being handled by various established GIS applications in Malaysia. Five established GIS applications namely eKadasOnline, SKiP, iPlan, TM SmartMap and DBKL Interactive Portal were selected and studied to investigate the adoption of NDCDB in the respective GIS applications. These GIS applications were chosen based on the fact that the software applications are owned by distinguished organisations with a large clientele, which promotes spatial enablement. Detail descriptions of the GIS applications are as shown in Table 1. It should be highlighted that the study was conducted with the notion that the positional spatial accuracy of the NDCDB shall be accepted as claimed by the Department of Survey and Mapping Malaysia (JUPEM) who is also the authorised data custodian for the cadastral survey database of Peninsular Malaysia and Federal Territory Labuan. Furthermore, upgrading initiatives are still ongoing to enhance its spatial accuracy (Jamil, 2017) and therefore is excluded from the study scope.

\section{MULTI-CASE STUDY METHODOLOGY}

Yin (2013) case study principles were adopted in this study that included; i) defining case-study propositions and rival explanations; ii) clear research questions; iii) case-study protocol; iv) linking data to propositions; and v) determine the criteria to interpret the findings. The overall multi-case study methodology is shown in Figure 1. The case-study was propositioned based on Statement 2, NDCDB's significant role in the land-based analysis that stated: "NDCDB can help provide the required result of a land-based spatial analysis when users understand its characteristics and know how to adopt it for spatial analysis correctly 


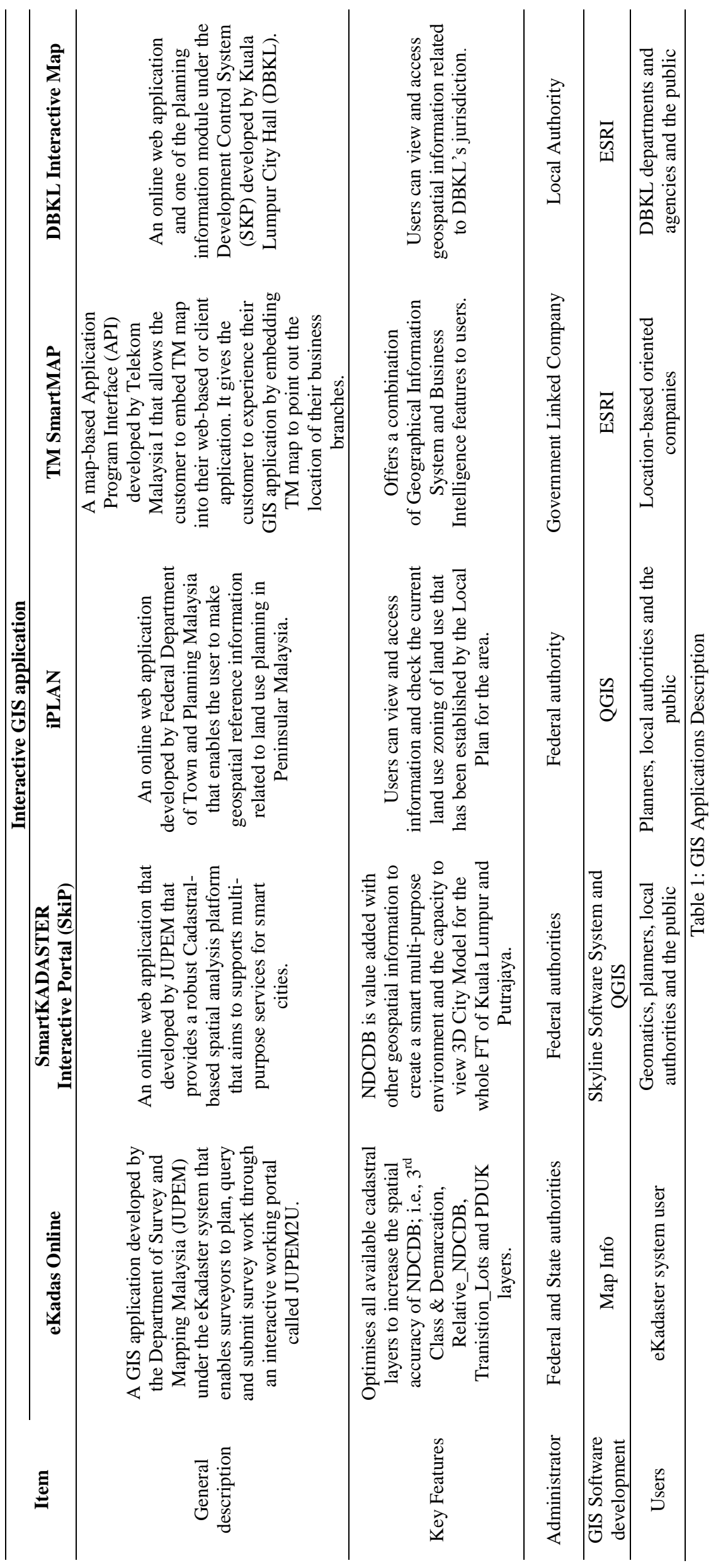




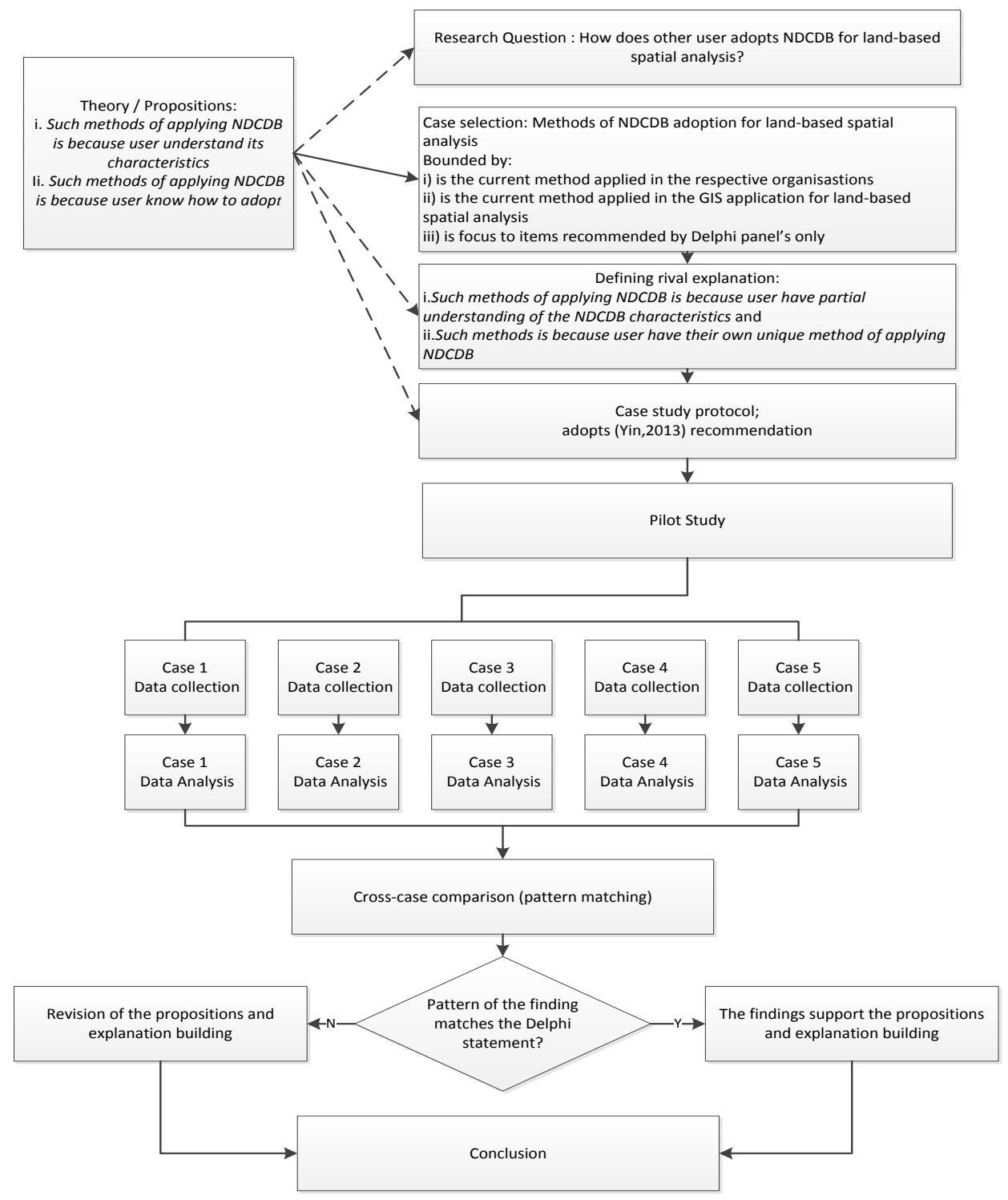

Figure 1: Multi-case study research methodology

The Delphi technique was used to establish Statement 2, NDCDB's significant role in the land-based analysis and was elaborated in detail in their paper by Halim et al. (2017). Consequently, adjustment was made on the statement to ease the case-study approach and was determined as; i) "Such methods of applying $N D C D B$ are because user understands its characteristics"; and ii) "Such methods of applying NDCDB is because users know how to adopt it." Meanwhile, the rival explanations were distinct as i) "Such methods of applying $N D C D B$ is because the user has a partial understanding of the NDCDB characteristics"; and ii) "Such methods are because the user has their own unique method of applying NDCDB." Propositions and rival explanations helped form the design of the case study conducted so attention can be given to specific data and unrelated data can be ignored (Yin, 2013). Construct validity, internal validity, external validity and reliability of the multi-case study were considered throughout the study.

Cross-case comparison analysis and explanation building were used to pattern matched and analyse the case-study data as suggested by Yin (2003). The case-study primary data came mostly from the GIS applications documentation, archived records and reports. Apart from that, the interview and general observation data were treated as secondary data to complement the primary data and support the findings of the document analysis to allow explanation building. According to Stake (2005), direct interpretation is considered a form of analysis, and for that, direct answers from the interviewee were analysed and interpreted directly in this study.

\section{ANALYSIS}

\subsection{Cross-case comparison analysis}

A visualisation technique of case-ordered descriptive metamatrix was used to showcase the cross-case comparison analysis as recommended by Khan and VanWynsberghe (2008). Table 2 shows the descriptive meta-matrix of the multi-case study conducted. The descriptive meta-matrix included all five unit analysis of Data Collection Matrixes and was structured and indexed into unit analysis/cases and variables. 


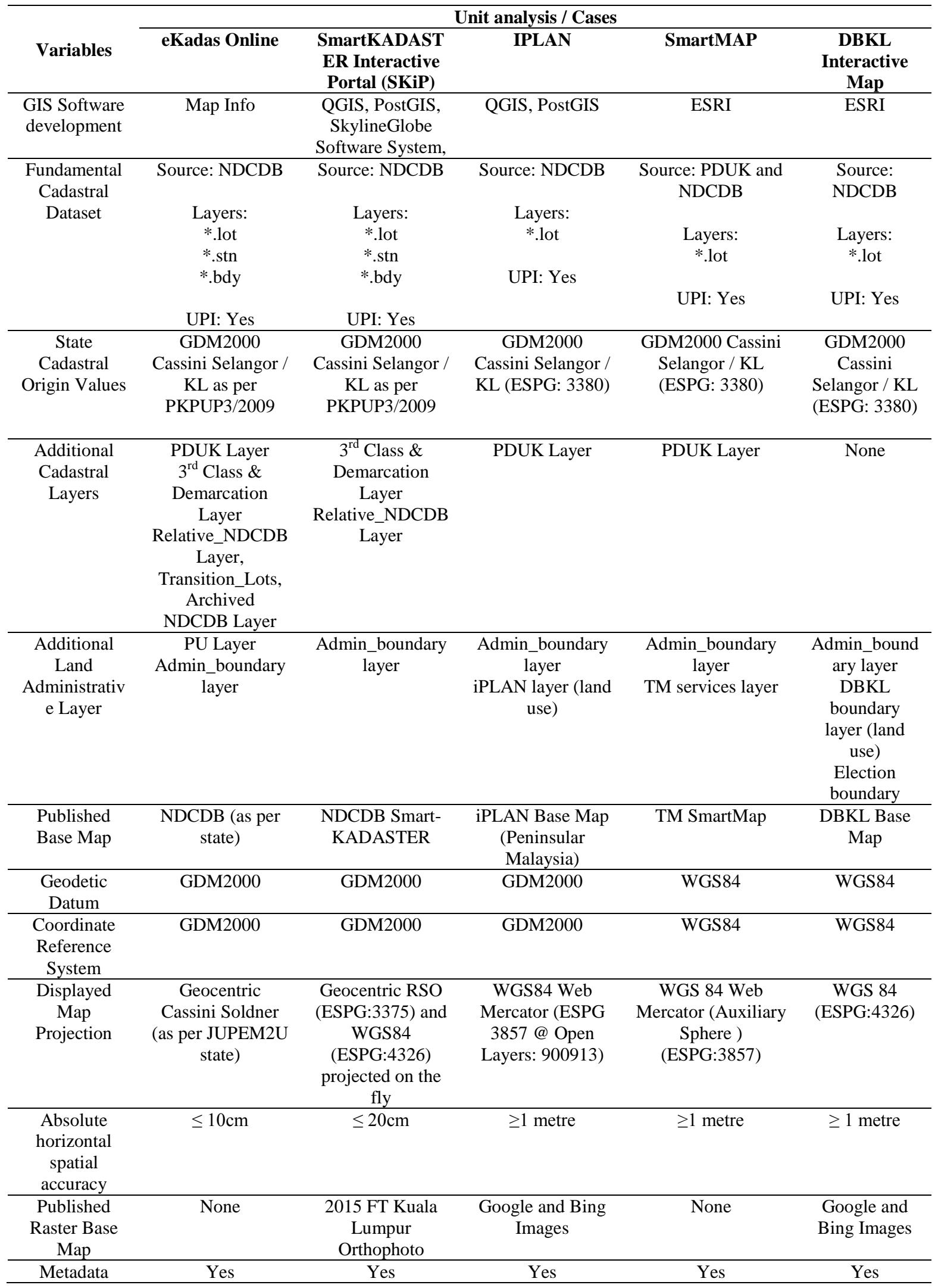

Table 2: Descriptive Meta-Matrix of the Multi-Case Study 
The determination of the variables is not highlighted in this paper but further explained separately in another paper. However, as an overview, the variables were identified by using the Delphi technique where 14 SMEs were involved, and consensus of the variables was achieved during Round 3. Aside from that few relevant variables were added based on the findings during the case-study pretesting.

In general, the flow of the NDCDB data handlings were similar by all GIS applications as shown in Table 2, but there were noticeable differences of handling the NDCDB between the GIS applications that included; i) the map projection parameters; ii) the geocentric datum used; iii) the obtained NDCDB layers and its additional layers; and iv) web map publishing display.

\subsection{The explanation for rival findings of NDCDB handling}

The notable findings of the cross-case comparison analysis and explanation building that led to the rival conclusion were; i) some users opt to utilise the map projections built in the GIS software because they assume the parameters were the same as the authorised and official ones from JUPEM - the action was justified as existing practice and users were not aware of the consequences; ii) some users were not aware the cadastral data stored in NDCDB were based on GDM2000 datum with ITRF2000 epoch 2006 and not epoch 2009; iii) some users assumed the *.lot layer was sufficient for spatial analysis and are not aware of the other NDCDB layer formats or the layer's relationship; iv) some users were mindful of the incompleteness of land parcels in the NDCDB, however, were unaware of the additional NDCDB layers that can complement the missing land parcels. The users instead opt to self-rectification by digitising another source of data to complement the missing land parcels, and lastly, v) existing practice of storing or displaying the geospatial data in WGS84 by some users were controversial. Users were not aware that different projection of WGS84 (Mercator vs Web Mercator) might result in the different result of the spatial analysis, especially in distance measurement.

\subsection{Recommendations based on evidence}

Since NDCDB is the product of the eKadaster system, naturally eKadasOnline was set as a benchmark in the event where rival findings occurred. The rival findings do not imply the methods chosen by users to be entirely correct or wrong for its application purposes but create an opportunity for further investigation on the subject. Therefore rectifying explanations or recommendations are justified for rival findings based on the benchmark, other cases best practices along with current policy implementations.

\subsubsection{Cut-Off-Date}

Cut-off-date is crucial information to determine the NDCDB status and currentness. The NDCDB can be downloaded instantly with the accuracy update of 24 hours. Even though changes may not occur as often, but the cut-off-date information can help explain a faulty occurrence such as; i) the 'incompleteness' of the data; ii) the spatial accuracy of the data; or iii) any erroneous implication that may have happened during the timeline, for example, a time where incomplete UPI characters were identified. The information can be supplied to JUPEM to track and determine for further data mitigation.

\subsubsection{Complete NDCDB Layer Format}

*.lot is commonly requested by users. It primarily provides the land parcel information required for land-based spatial analysis. However, by including *.bdy and *.stn, holistic spatial analysis can be conducted that include adjacent boundary query and query lots by coordinate. Erroneous map projection can also be avoided when boundary mark's coordinate information is available for map comparison.

\subsubsection{State Cadastral Origin Values}

\section{i. Authorised Parameters}

It is advisable for users to user-defined the state cadastral origin values as per described in the PKPUP $3 / 2009$ (JUPEM, 2009) when reprojecting NDCDB to another GIS software or platform. Table 3 shows the different Bukit Asa origin values from various sources. Built-in projections in the off-the-shelve or open-sourced GIS software may claim to have the correct projection of Geocentric Cassini Selangor / KL, but investigation carried out during the study implied otherwise. Built-in projections from two primarily used off-the-shelve GIS software, namely MapInfo Professional version 11 and ESRI ArcGIS version 10.3, and an open-sourced GIS software namely QGIS Version 2.18.15 were compared over the parameter values described in PKPUP 3/2009, specifically the GDM2000 values which were actually a revised version of GDM2000 conducted and aligned to ITRF2000 at epoch 2006. It was found out that QGIS and ESRI ArcGIS used the ESPG:3880 parameter values, while MapInfo Professional optimised the GDM2000(2009) Cassini Soldner parameter values. The usage of non-authorised parameter values may in result provide inaccurate spatial placement, thus hinders accurate spatial analysis that the native NDCDB stored in JUPEM is projected to the GDM2000 (2006) datum.

\begin{tabular}{ccccc}
\hline Item & Latitude & Longitude & $\begin{array}{c}\text { Arc length distance } \\
\text { difference (km) from } \\
\text { GDM2000 origin values }\end{array}$ & Remark \\
\hline PKPUP 3/2009 & 3.68010494 & 101.50680161 & 0 & Values for GDM2000 \\
\hline PKPUP 3/2009 & 3.68010486 & 101.50680036 & 0.00013899 & $\begin{array}{c}\text { Values for GDM2000 } \\
(2009)\end{array}$ \\
\hline $\begin{array}{c}\text { MapInfo Professional } \\
\text { Version 11 }\end{array}$ & 3.68010494 & 101.50680161 & 0.00013899 & $\begin{array}{c}\text { In accordance to PKPUP 3/2009 but } \\
\text { expressed in GDM(2009) }\end{array}$ \\
\hline ESRI ArcGIS 10.3 & 3.68464905 & 101.38910791 & 13.06969418 & According to ESPG:3380 \\
\hline QGIS 2.18.15 & 3.68464905 & 101.38910792 & 13.06969308 & According to ESPG:3380 (released 16 \\
October 2017)
\end{tabular}

Table 3: Bukit Asa's Origin Value Differences 


\section{ii. Adopted GDM2000 in NDCDB}

NDCDB is projected to GDM2000 or GDM2000 conducted in epoch 2006 and not GDM2000(2009) due to different reason and discrepancy of the GDM2000-based database (Shariff et al., 2017; Shariff et al., 2014) that may implicate legal concerns. On that note, it should be recognised that different map projection parameters of the NDCDB projection is inevitably to cause spatial displacement or Arc length distance difference of at least $13 \mathrm{~km}$ as shown in Table 3.

\section{iii. Discarding False Easting and False Northing}

The False Easting and False Northing is an imaginary grid coordinate system that translated the $\mathrm{X}, \mathrm{Y}$ constants from a true origin to a false origin assigned to the true origin of the grid system. Historically, it was used by surveyors to extend farther positive value coordinates to the zone assigned to false grid origin for computational purposes (Snyder, 1987). Most of the state's true origins are not explicitly defined (Shariff et al., 2014) but mentioned offsets assuming from central meridians. However, False Easting and False Northing do not affect the look of the coordinate by distorting the map due to its application and usually discarded for analysis when the geocentric datum is adopted (Hooijberg, 2007). This is also in line with the parameters set in PKPUP 3/2009 where the False Easting and False Northing are both 0,0 (JUPEM, 2009b). The latitude and longitude of the origins are not referred to a single triangulation system but to three different triangulation systems, namely, PERAK, ASA and MRT Systems (Nordin, 2001). In result, the ESPG:3880 having the values of False Easting and False Northing is deemed irrelevant.

\section{iv. Spatial Displacement}

Based on Table 3, the arc-length distance between ESPG:3380 origin values (IOGP, 2017) and the official GDM2000 parameter in PKPUP3/2009 origin is approximately $13 \mathrm{~km}$. However, misinformed users will experience an error of $60 \mathrm{~km}$ in grid distance displacement. Figure 2 shows the horizontal spatial displacement of ESPG:3380 and user-defined Geocentric Cassini Selangor / Kuala Lumpur map projection parameters using MapInfo Professional Version 11. The displacement occurred when polygons from these two map projections were loaded into MapInfo Professional Version 11. The software was unable to perform the correct reprojection resulting in the two polygons not positioned on top of each other. Instead, it showed horizontal spatial displacement as seen in Figure 2. However, for unknown reasons, MapInfo managed to show the correct geodesic distance of $13 \mathrm{~km}$. Figure 3 , on the other hand, shows vertical spatial displacement of ESPG:3380 and userdefined Geocentric Cassini Selangor / FT Kuala Lumpur map projection using both QGIS Version 2.18.15 (Left) and ArcGIS 10.3 (Right). Both of the GIS software successfully reprojected the polygons and was able to position the polygons on top of each other by deleting the PRJ files and reloading the layers into arbitrary Cartesian coordinates. However, it is here that a distance of $60 \mathrm{~km}$ spatial displacement between the two occurred as shown in Figure 3. Therefore, by any circumstance the builtin map projection parameters or ESPG:3380 is being used, horizontal and vertical spatial displacement will surely occur in all three software due to different parameters applied.

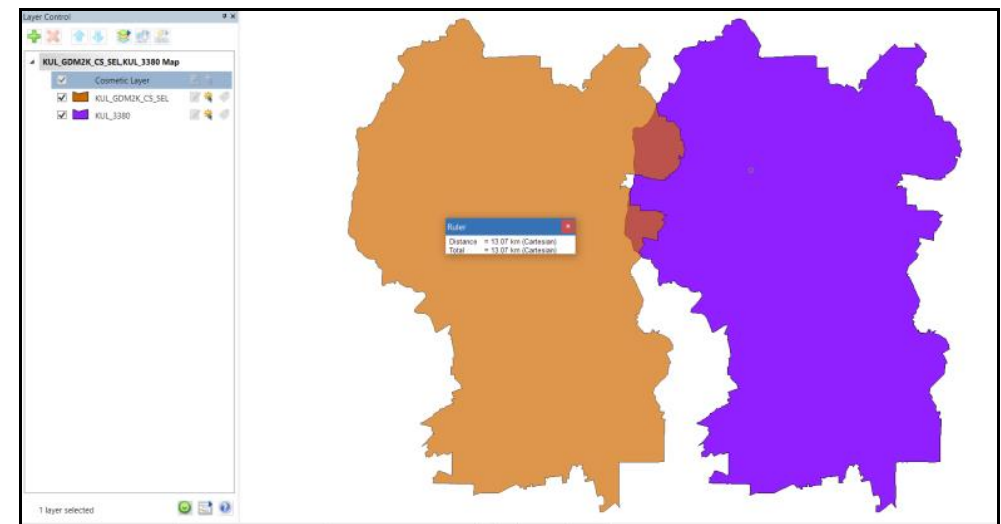

Figure 2: Horizontal Spatial Displacement of ESPG:3380 and User-Defined Geocentric Cassini Selangor / Kuala Lumpur Map Projection Parameters Using MapInfo Professional Version 11

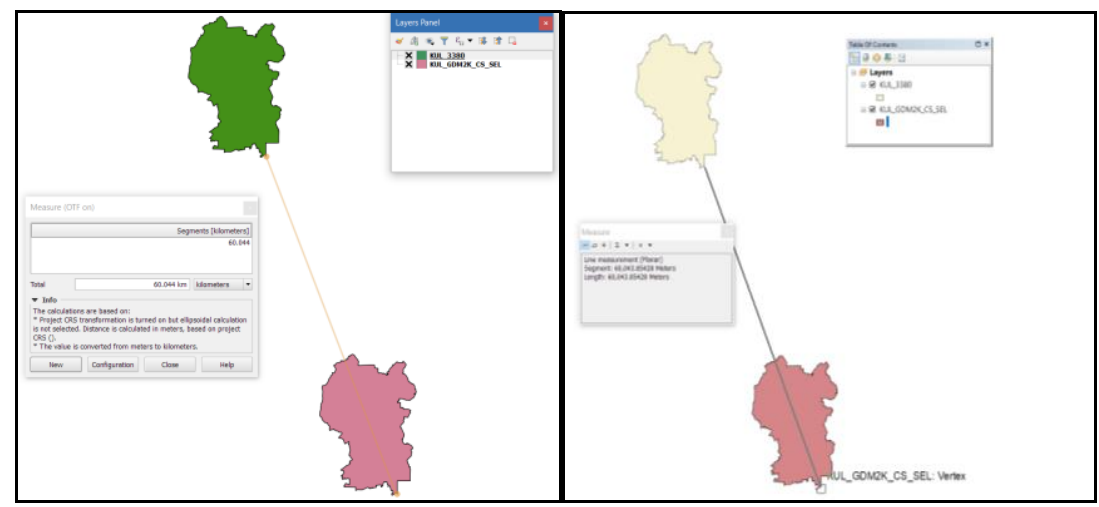

Figure 3: Vertical Spatial Displacement of ESPG:3380 and User Defined Geocentric Cassini Selangor / FT Kuala Lumpur Map Projection Using QGIS Version 2.18.15 (Left) and ArcGIS 10.3 (Right) 


\subsubsection{Additional Cadastral Layers}

NDCDB was described as incomplete during the case study, and in result, the densified lots of the previous DCDB (or PDUK) was highly complimented by users. Unfortunately, PDUK was no longer maintained, and the last update took place in 2010. The incompleteness of the NDCDB was the result of incomplete spatial information of adjacent sub-class lots, blunder errors of previous data such as; i) sub-class survey lots were accidentally included into the NDCDB adjustments; or ii) mistakes made from keyboard entering during the development of PDUK. Therefore the related lots within the NDCDB block that do not meet the minimum spatial tolerance and weightage are not appended to NDCDB, thus creating the missing lots.

To overcome some missing land parcels, it is suggested for additional NDCDB layers to be obtained as well. The additional NDCDB layers namely 3rd Class and Demarcation Survey, Transition_Lots, Relative_NDCDB and Archived_NDCDB are basically final surveyed land parcels. Therefore the accuracy of the spatial information is at least equivalent to the minimum cadastral survey data accuracy. Figure 4 shows the differences between an 'incomplete' NDCDB versus a complete NDCDB that have been complemented with the other additional NDCDB layers. The complete NDCDB with additional NDCDB layers are more populated and gives a holistic view of the area of interest. Better and accurate decision making can be conducted when the NDCDB lots are densely populated. Although these additional layers are a temporary database, the lots will eventually be appended to the primary NDCDB from time to time (Jamil, 2017) with better spatial accuracy. Its presence accommodates user for a better and accurate land-based spatial analysis rather than an incomplete NDCDB or going through tedious work of 'updating' or digitising the missing NDCDB with questionable spatial accuracy.

\section{RESULT}

Based on the evidence of the multi-case study, the rival propositions were found most suitable to summarise the overall findings, which was such methods of applying NDCDB for land-based spatial analysis were because some users (GIS application administrator and developer) have a partial understanding of the NDCDB characteristics. Therefore, such methods were applied by some users according to their own conception and suitability. The findings were concluded based on the cross-case comparison analysis findings and explanation building process. Direct observations also concurred with the findings where it was observed that; i) $60 \%$ of the users have apparently limited knowledge on handing NDCDB data; ii) half of the GIS application administrator relied on the developers or operators for technical related rival explanations; iii) except for SKiP, there was no significant guideline that users can refer to when handling NDCDB data or other geospatial data; iv) GIS software literacy issue is a concern as users tend to rely on experienced operators based on previous or existing practices in the organisations; and v) except for eKadasOnline, NDCDB was used one-off during the GIS application development stage and was updated manually.

\section{DISCUSSION AND CONCLUSION}

It was well understood from the multi-case study that different users might call for different requirement because of various clientele. However, to achieve accurate land-based spatial analysis the fundamental data source, which is the NDCDB should be at least handled correctly. The development of any cadastral database in the world is costly and required considerable time and resource for its development (Dale, 1976; Thompson, 2015). Therefore it is recommended that the NDCDB should be appropriately handled and optimised to avoid wasteful and exhausting resources.

Consequently, the multi-case study findings were imperative to have a particular stance on the application of NDCDB by various GIS applications. It was instead a surprising finding considering the GIS applications were of established organisations. However, this also implies the likeliness that other casual GIS application developers may have also practised the same way.

Some possibilities may be explicated by the rival findings found in this study. For instance, it was noticeable that lack of awareness programs from the relevant authorities have contributed to the limited knowledge and exposure to NDCDB, besides the unavailability of aiding tools for user reference. Due to the absence of an aiding tool, strong dependency towards personnel or GIS application operators who have had experience in data handling or possess supposing knowledge of previous or 'assumed'best-practices transpired to the rival findings.

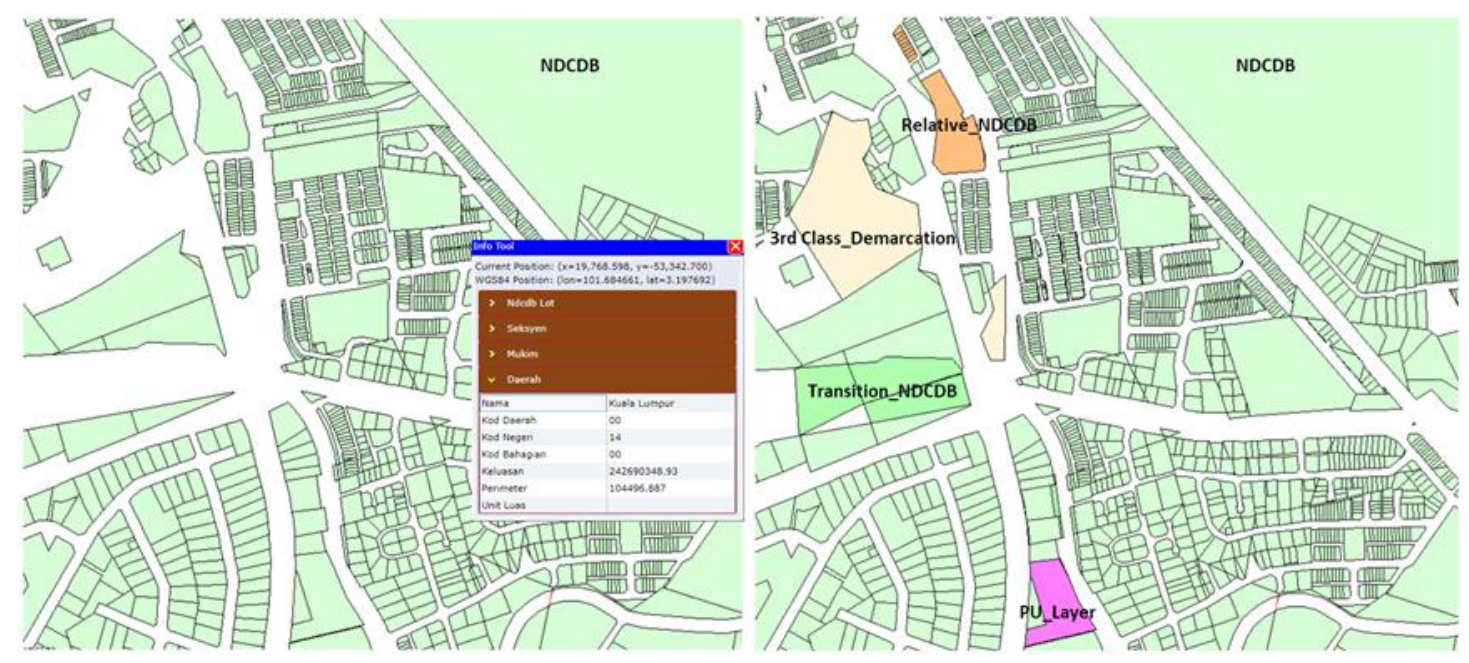

Figure 4: Sample of 'Incomplete NDCDB' (Left) vs 'Completed NDCDB' (Right) With Additional NDCDB layers 
Other possibilities to explain the rival findings would be the request for NDCDB updates were not on a regular basis, and NDCDB was obtained one-time, implying the process of NDCDB data handling seldom occurred. In the event where the users felt the NDCDB was obsolete, applications for a new NDCDB version was made. This method appeared to be impractical considering NDCDB can be retrieved almost realtime (Jamil, 2017). The existence of feature map services with the advent of service-oriented architecture could very well be suggested to allow the real-time NDCDB update to users.

Based on the above possibilities, striking conclusions can be made on top of the multi-case study rival propositions; i) the knowledge of NDCDB handling for land-based spatial analysis was not well distributed and ii) rectifying knowledge-based mistakes are deemed necessary. Hence, recommendations for NDCDB data handling from this study should be considered to enable full optimisation of NDCDB for multipurpose usage, especially for land-based spatial analysis.Other than that, the study has summarised that full potentials of NDCDB can be tapped by; i) optimising all the NDCDB layer formats for a holistic spatial analysis result; ii) optimising all the NDCDB's additional layers to complement the 'missing' or 'incomplete' land parcels; iii) optimising NDCDB spatial accuracy of centimeters level; iv) optimising the spatial existence of NDCDB's additional layers for planning purposes; and v) the NDCDB is updated almost real time, making the data is relevant to the cut-off-date. Evidently, data replication, exhaustion of resources, and reduce risks or costly investments made by decision makers, policy makers, developers or individuals can be avoided when NDCDB is fully optimised to assist decision making.

\section{CONTRIBUTION}

This study has contributed to the existing body of knowledge in understanding the NDCDB. Previously, there were limited empirical data available on unfolding the adoption of NDCDB by various GIS application users. This research has identified recommendations to rectify knowledge-based mistakes related to NDCDB data handling and means to fully tapped the NDCDB potentials. The outcome of this research will aid land surveyors, land administrators, and GIS users (professional or casual), as well as the general public, to properly handle NDCDB for multipurpose usage. With the result and recommendations emplaced, it is hoped the usage of NDCDB increases to encourage spatially accurate analysis result that also include the disaster management domain.

\section{ACKNOWLEDGEMENTS}

The authors would like to thank Universiti Teknologi MARA, JUPEM, and all related agencies for providing valuable information for this study. Thanks are also due to all my colleagues who assisted during this study data and last, but not least the unknown reviewers are gratefully acknowledged.

\section{REFERENCES}

Ahmad, S. (2014). GIS (Geographical Information System) Implementation in Public Sector. (Masters), University Technology Mara, Shah Alam.

Chan, K.-T. (2016). Introduction to Geographic Information Systems (8th ed.). New York: McGraw Hill Education
Dale. (1976). Cadastral Surveys Within the Commonwealth: Report: H.M. Stationery Office.

Dale, M. R., \& Fortin, M.-J. (2014). Spatial analysis: a guide for ecologists: Cambridge University Press.

Halim, N. Z. A., Sulaiman, S., Talib, K., Yusof, O., Wazir, M., \& Adimin, M. (2017). Identifying the role Of National Digital Cadastral Database (NDCDB) in Malaysia and for land-based spatial analysis. International Archives of the Photogrammetry, Remote Sensing \& Spatial Information Sciences, 42.

Halim, N. Z. A., Sulaiman, S. A., Talib, K., \& Ng, E. G. (2018). Identifying the relevant features of the National Digital Cadastral Database (NDCDB) for spatial analysis by using the Delphi Technique. IOP Conference Series: Earth and Environmental Science, 117(1), 012030.

IOGP. (2017). ESPG Geodetic Parameter Data Set [Paramater]. ESPG:3880.

Jamil, H. (2017) Role of the Department of Survey and Mapping Malaysia (DSMM) on the cadastral survey and the significance of National Digital Cadastral Database (NDCDB) to the nation/Interviewer: N. Z. A. Halim. Buletin Geospatial Sektor Awam Tahun 2017, MaCGDI, Putrajaya.

JUPEM. (2009). Garis Panduan Mengenai Penukaran Koordinat, Transformasi Datum Dan Unjuran Peta Untuk Tujuan Ukur Dan Pemetaan. (Pekeliling Ketua Pengarah Ukur \& Pemetaan 3/2009). Kuala Lumpur: JUPEM.

Khan, S., \& VanWynsberghe, R. (2008). Cultivating the undermined: Cross-case analysis as knowledge mobilization. Paper presented at the Forum Qualitative Sozialforschung/Forum: Qualitative Social Research.

MACGDI. (2017). Transaksi Data NDCDB melalui MyGEOPORTAL daripada tahun 2010 - 2017

Shariff, Gill, J., Amin, Z., \& Omar, K. (2017). Towards the Implementation of Semi-Dynamic Datum for Malaysia. International Archives of the Photogrammetry, Remote Sensing \& Spatial Information Sciences, 42.

Shariff, Musa, T. A., Omar, K., \& Othman, R. (2014). The Geocentric Datum of Malaysia: Preliminary Assessment and Implications Geoinformation for Informed Decisions (pp. 7183): Springer.

Stake, R. E. (2005). Qualitative case studies. In N. K. Denzin \& Y. S. Lincoln (Eds.), The SAGE handbook of qualitative research (3rd ed., pp. 443 - 466). Thousand Oaks, CA: SAGE Publications.

Thompson, R. J. (2015). A model for the creation and progressive improvement of a digital cadastral data base. Land Use Policy, 49, 565-576. doi:http://dx.doi.org/10.1016/j.landusepol.2014.12.016

Yin, R. K. (2003). Case study research: Design and methods (Second ed.): SAGE publications.

Yin, R. K. (2013). Case study research: Design and methods (Fifth ed.): SAGE publications.

Revised August 2018 